\title{
Tunable Zero-Index Photonic Crystal Waveguide for Two-Qubit Entanglement Detection
}

\author{
Ege Özgün, ${ }^{* \dagger}$ Ekmel Ozbay, ${ }^{\dagger, \ddagger, \S}$ and Humeyra Caglayan ${ }^{*}{ }^{\dagger}$
}

${ }^{\dagger}$ Nanotechnology Research Center, ${ }^{\ddagger}$ Department of Physics, and ${ }^{\S}$ Department of Electrical and Electronics Engineering, Bilkent University, 06800 Ankara, Turkey

\section{Supporting Information}

ABSTRACT: Preparation and measurement of an entangled state are essential in quantum information applications. The recent theoretical works on preparing and measuring entangled states via coupled quantum dot-waveguide platforms are promising. However, an alternative strategy is still needed for more accessible implementations. In this Article, we propose a simple but powerful medium for quantum information processing and communication consisting of a zero-index photonic crystal (PC) waveguide (WG). By exploiting the zero-index behavior of the PC WG, we overcome the difficulty of sensitivity in quantum dots' location, which is one of the main challenges for preparing and measuring a twoqubit entangled state. Moreover, our platform possesses the feature of tunability, which allows it to operate in different wavelength regimes. The suggested medium is also promising for realizing long-range spatial

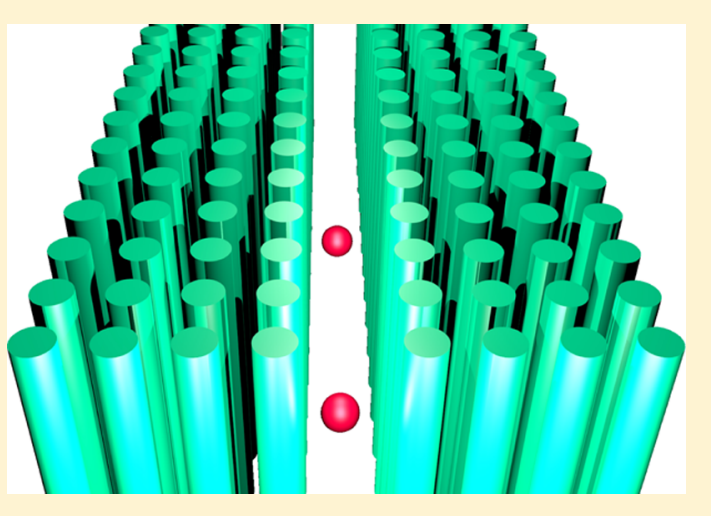
coherence as well as long-range entanglement.

KEYWORDS: quantum entanglement, photonic crystals, zero-index waveguide

uantum entanglement is one of the most counterintuitive outcomes of quantum mechanics and is central in quantum information science. ${ }^{1}$ It is the leading actor in various phenomena such as quantum teleportation, ${ }^{2,3}$ quantum cryptography, ${ }^{4}$ and quantum computation. ${ }^{5}$ It is essential to quantify the entanglement for a systematic study of the quantum information platforms. There exists different strategies for defining the degree of quantum entanglement. Concurrence $^{6}$ is one of the most commonly used entanglement measures, which we will also utilize through this paper.

There is a tremendous effort to devise a method for consistently preparing and measuring entanglement. ${ }^{7,8}$ An experimental work in 2010 utilized a superconducting circuit to prepare and measure three-qubit entanglement. ${ }^{9}$ The motivation behind obtaining a multiqubit entangled state is its necessity for quantum error correction schemes, which starts with tripartite entanglement. The realization of multiqubit entanglement was previously achieved for spins, ${ }^{10}$ ions, ${ }^{11}$ and photons. ${ }^{12}$ There also exists numerous studies utilizing WGs to achieve entanglement. ${ }^{13-16}$ Despite these efforts, a more robust and accessible strategy is still necessary for another step forward in quantum information, since although the above-mentioned strategies are successful, it would be dramatically difficult to integrate them into a quantum information device. Quantum dots (QDs) play a significant role in quantum computation ${ }^{17}$ and can be integrated into waveguides (WGs), which are quite accessible. Therefore, to overcome these problems, a WG-QD combined platform is a very promising candidate. There are two recent proposals that use WG-QD coupled schemes. The first one utilizes a plasmonic WG operated near its cutoff frequency to attain epsilon near-zero (ENZ) behavior, ${ }^{18}$ whereas the other one uses the correspondence between twophoton detection probability and concurrence, which is a manifestation of the symmetry obtained by forcing the QDs to interact at the origin. ${ }^{19}$ Although those are powerful strategies, a tunable and more accessible alternative would be of high interest for robust quantum information applications.

There are also two recent proposals for controlled coupling of photon emitters: The first one is a nanowire-based photonic crystal (PC) WG system, which shows a modified Mollow triplet spectrum, ${ }^{20}$ and the second is a three-dimensional polariton WG structure. $^{21}$

We propose a platform based on the above-mentioned twophoton detection probability scheme and enhance it by using a tunable platform of a PC WG operated near its cutoff frequency, which yields a zero mode index for the WG. These materials are referred to as ENZ materials. ${ }^{22}$ Among different ways of realizing such materials, ${ }^{23}$ the most straightforward one is exploiting the dispersion of the WG by operating near its cutoff frequency. ${ }^{24,25}$ It is important to note that although our platform effectively operates within the ENZ region, we are not working in the ultrastrong coupling regime, in which even the rotating wave approximation can break down. $^{26}$

Received: August 9, 2016

Published: October 17, 2016 
$\mathbf{a}$

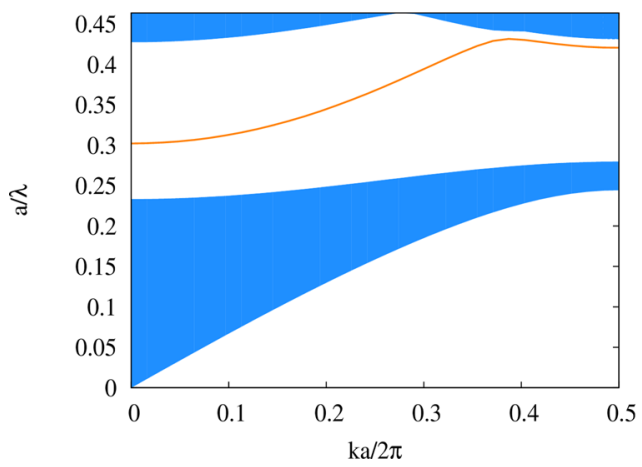

b

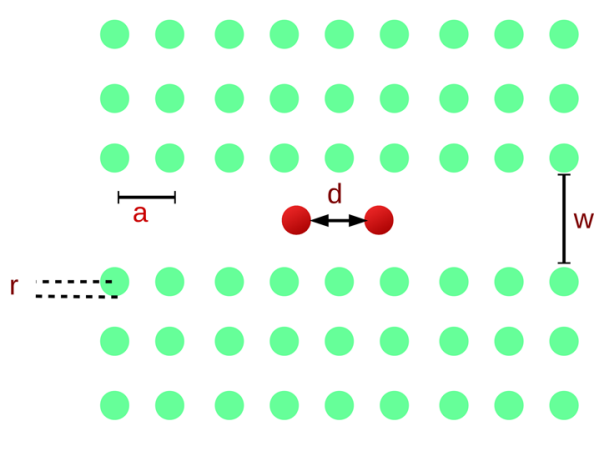

Figure 1. (a) Dimensionless frequency $a / \lambda$ plotted versus the dimensionless mode index $k a / 2 \pi$, depicting the disallowed regions, photonic band gaps, and the guiding mode. (b) Top view of the WG-QD coupled system with an illustration of significant parameters.
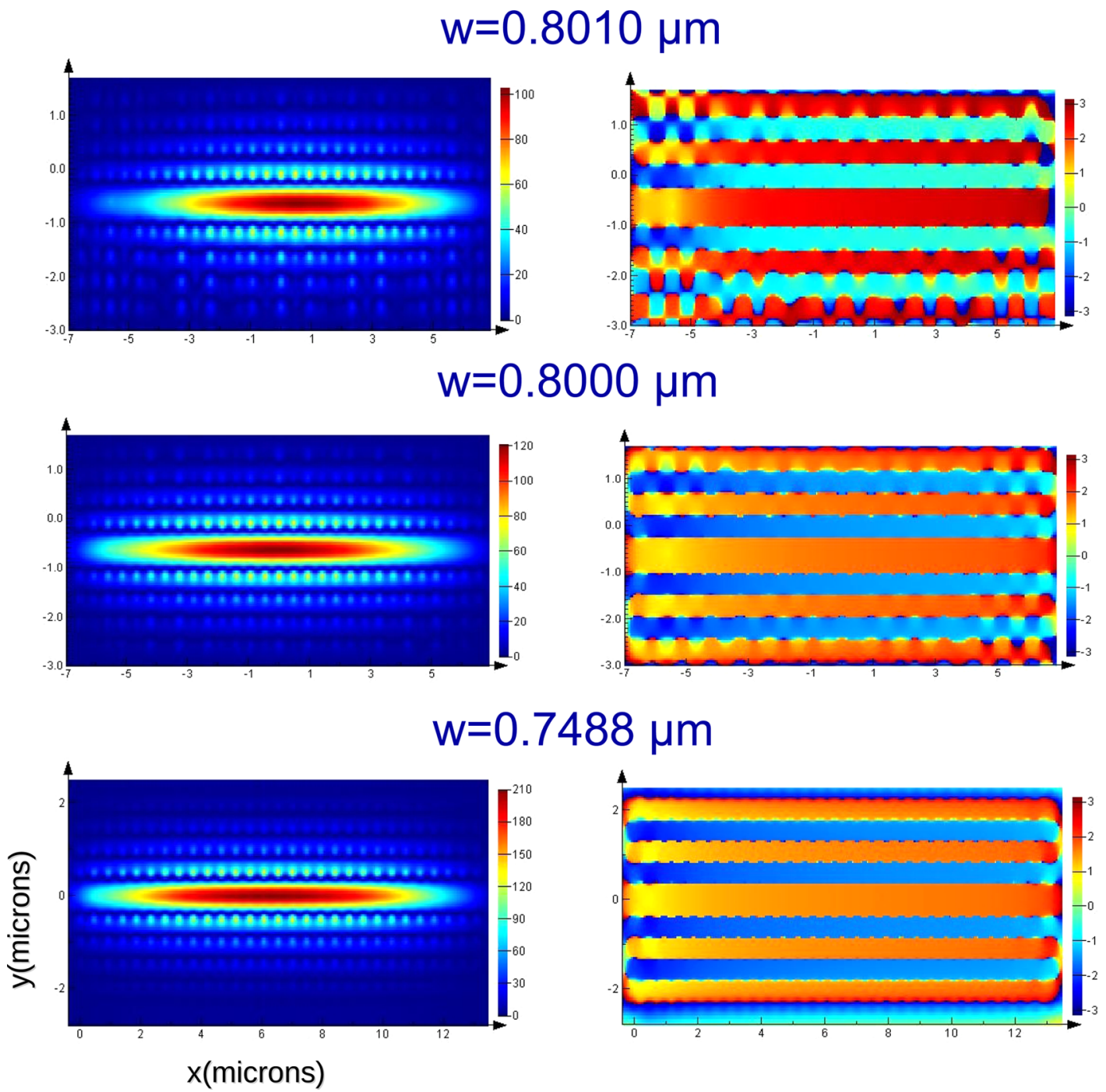

$\mathrm{w}=0.7488 \mu \mathrm{m}$

Figure 2. Magnitudes and phases of the E-field for the InP PC WG for different WG widths $(w)$. From top to bottom, the zero-index behavior is obtained for the wavelengths $1.55140,1.54297$, and $1.48341 \mu \mathrm{m}$, respectively. The $w=0.7488 \mu \mathrm{m}$ case corresponds to removing a single line of rods. By changing $w$, we can tune the wavelength for which zero-index behavior is observed.

The ENZ medium stretches the effective wavelength inside the structure, and as a result the distance $(d)$ between the sources and/or scatterers, although physically sizable, becomes electrically small compared with the effective wavelength $\left(\lambda_{\text {eff }}\right)$.
In other words, here $d / \lambda_{\text {eff }}$ becomes small, not because $d$ is small, but because $\lambda_{\text {eff }}$ becomes very large due to the effect of the ENZ medium. As a result, ENZ materials offer several exciting potential applications, such as supercoupling and 
$\mathbf{a}$

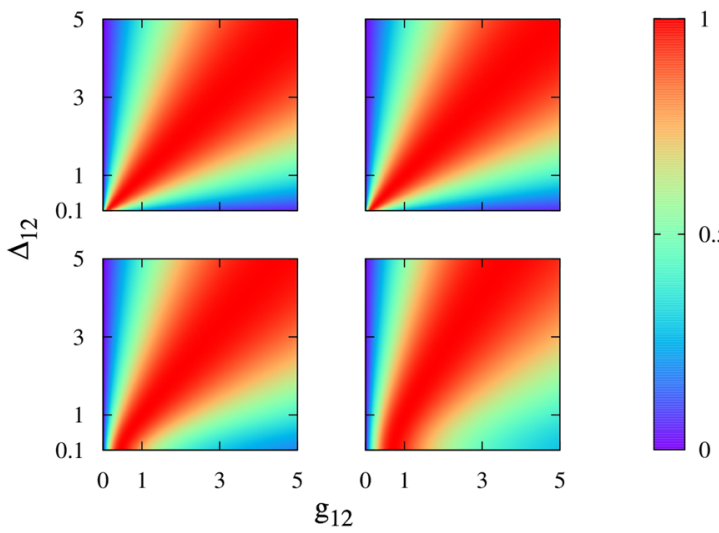

b

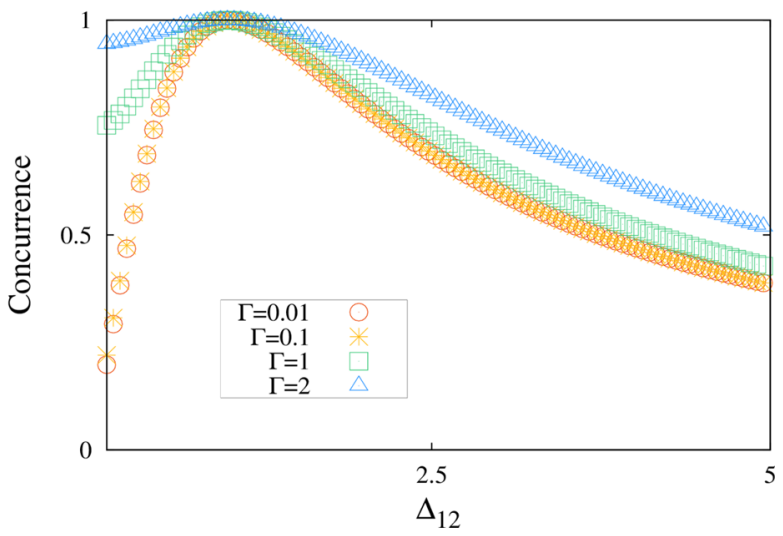

Figure 3. (a) Concurrence shown for $\Gamma=0.01$ (top left), 0.1 (top right), 1 (bottom left), and 2 (bottom right) versus $g_{12}=g_{1} / g_{2}$ and $\Delta_{12}=\Delta_{1} / \Delta_{2}$. (b) Slices from the left panel plotted for $g_{12}=1$, which is required to obtain the correspondence between two-photon total detection probability and concurrence that the measurement strategy is based on.

tunneling, ${ }^{22}$ photon density of states enhancement, ${ }^{27}$ directive emission, ${ }^{28}$ enhanced transmission, ${ }^{29}$ and radiation phase front shaping. ${ }^{30,31}$ Instead of having cavities that enhance the electric field (E-field) in a subwavelength area with limited effective mode areas, ${ }^{32}$ the suggested nanophotonic devices will effectively stretch the effective mode to the whole structure. In homogeneous electromagnetic media, the wavelength defines the spatial scale, and a very large wavelength implies a very low frequency. However, in an ENZ medium, the WG mode has an extremely extended wavelength, while its frequency lies within an optical or telecom band. Therefore, an ENZ medium creates an environment where the interaction is emitter position insensitive. Here, we propose an all-dielectric PC WG as an ENZ medium for quantum information applications, for the first time. The suggested platform is accessible in terms of both its fabrication and the excitation of the quantum emitters inside the WG.

PCs are ideal platforms for achieving confinement and guided transmission of electromagnetic waves. Moreover, they give rise to numerous interesting phenomena including negative refraction, ${ }^{33}$ slow waves, ${ }^{34}$ and nonreciprocal chirality and asymmetric transmission. ${ }^{35}$ In this Article, we suggest an InP-based PC WG as a platform for preparing and measuring a two-qubit entangled state. To obtain the suggested PC WG, a line defect is opened in the middle of the square lattice of cylindrical InP rods with radii $r=0.2 a$, $a$ being the lattice constant of the PC. The fabrication procedure of the InP rods is described in ref 36. The band structure of the InP PC WG is given in Figure 1a, and relevant parameters are sketched in Figure $1 \mathrm{~b}$.

Figure 2 illustrates the zero-index regimes obtained for different WG widths $(w)$. Left panels show the magnitude of the E-field, whereas the constant phases shown in the right panels are the clear signatures of the zero-index behavior. By varying $w$, it is possible to obtain zero-index platforms operating in different wavelengths, which is one of the significant advantages of our proposal. The $w=0.8010 \mu \mathrm{m}$ case gives a zero-index WG for $\lambda=1.55140 \mu \mathrm{m}$, which is quite favorable, since most of the detectors work in the vicinity of $1.55 \mu \mathrm{m}$. The spectral bandwidth of the ENZ regime is approximately $\sim 11$ $\mathrm{nm}$, which can be seen from the second figure given in the Supporting Information.
The other important component of the suggested platform is QDs. The incoming WG mode $\omega_{k}$ must be large enough to

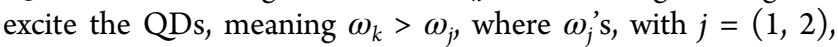
denote the transition frequencies of the QDs. Moreover, QD absorption frequencies must coincide with the cutoff frequency of the WG. Thanks to the zero-index behavior of the PC WG, the positions of the QDs are quite flexible, which is essential for the measurement procedure.

\section{THE MODEL}

We will treat the QDs as two-level systems, beginning with a Jaynes-Cummings-type Hamiltonian for the coupled WGQD system ${ }^{37-40}$ in the rotating wave approximation and take $\hbar$ $=1$ :

$$
\begin{aligned}
\hat{H}=\sum_{k}\left[\omega_{k} c_{k}^{\dagger} c_{k}+\sum_{j=1}^{2} V_{k}^{(j)}\left(c_{k}^{\dagger} \sigma_{-}^{(j)}+c_{k} \sigma_{+}^{(j)}\right)\right] \\
+\sum_{j=1}^{2} \sigma_{+}^{(j)} \sigma_{-}^{(j)}\left(\omega_{j}-i \frac{\Gamma_{j}}{2}\right)
\end{aligned}
$$

where $c_{k}^{\dagger} / c_{k}$ are bosonic creation/annihilation operators, $V_{k}^{(j)}$ 's are the photon-QD interactions, $k$ is the wave vector of the photon, $\sigma_{+}^{(j)} / \sigma_{-}^{(j)}$ are the raising/lowering operators for the QDs, and $\Gamma_{j}$ 's contain the dissipation effects, including the ohmic losses and losses to the WG. We will exploit the ENZ nature of the platform; hence we can place the QDs with any separation as long as $k \ll 1 / d$ is satisfied. Since we are operating our WG near its cutoff frequency, we can have sufficiently small values for $k$, which gives us the freedom in our choice of $d$. Therefore, we can safely assume that the QDs are interacting at the origin and yet at the same time they are distant enough so that we can neglect the direct interaction between them. In other words, mathematically, there is no difference between placing the QDs at the origin or placing them with any separation $d$, unless $k \ll$ $1 / d$ is violated. Zero-index behavior of the WG not only simplifies the algebra but also is essential for the measurement strategy, which is available for a certain symmetry configuration, that is obtained when the QDs are interacting at the origin. ${ }^{19}$ For our system, the concurrence is given by 


$$
C=2 g_{12} \frac{\sqrt{\left(\Gamma^{2}+4 \Delta_{12}^{2}\right)\left(\Gamma^{2}+4\right)}}{\left(\Gamma^{2}+4 \Delta_{12}^{2}\right)+g_{12}^{2}\left(\Gamma^{2}+4\right)}
$$

where the dimensionless parameters are defined as $\Delta_{12} \equiv \Delta_{1} /$ $\Delta_{2}, g_{12} \equiv g_{1} / g_{2}$, and $\Gamma \equiv \Gamma_{1} / \Delta_{2}$ with $\Delta_{1} \equiv \epsilon-\omega_{1}$ and $\Delta_{2} \equiv \epsilon-$ $\omega_{2}, \epsilon=\omega_{k}$ is the eigenenergy of the system, and $g_{1}$ and $g_{2}$ are the single-mode WG-QD interactions. Here equal losses from the QDs are assumed, i.e., $\Gamma_{1}=\Gamma_{2}$. Details of the derivation are given in the Supporting Information. Figure 3 summarizes the results of the analytical calculations for the concurrence. Concurrence is plotted against varying $g_{12}$ and $\Delta_{12}$ values for different values of $\Gamma$ in Figure $3 \mathrm{a}$. To focus on the condition $\left(g_{1}\right.$ $=g_{2}$ ) that is required for the measurement proposal, the $g_{12}=1$ slice is shown in Figure $3 \mathrm{~b}$.

\section{MEASUREMENT STRATEGY}

By defining odd and even operators, assuming equal photonwaveguide interaction strengths $\left(g_{1}=g_{2}\right)$, and recasting the Hamiltonian, one sees that the interaction term contains only even operators. By exploiting this result, the concurrence can be related to a physically measurable entity, ${ }^{19}$ namely, two-photon total detection probability $\left(P_{\mathrm{RR}}\right)$,

$$
\left|\frac{P_{\mathrm{RR}}(\xi)}{P_{\mathrm{RR}}(\xi=0)}-1\right|=\frac{2 \operatorname{Re}[\xi]}{1+|\xi|^{2}}=C
$$

where $\xi=\alpha / \beta$, with $\alpha$ and $\beta$ being the probability amplitudes for QD1 and QD2 to absorb a photon and make a transition from their ground to excited states, and $P_{\mathrm{RR}}(\xi=0)$ is a fully disentangled state. Two separate measurements are required to extract the concurrence information as eq 3 illustrates: The first one is needed to measure $P_{\mathrm{RR}}(\xi=0)$, whereas the second one is for the two-photon total detection probability for the entangled state, i.e., $P_{\mathrm{RR}}(\xi)$. The details of the measurement procedure is described in ref 19 . The advantage of our platform within the context of the measurement strategy is twofold: First it enables separation insensitivity for the QDs, which makes the measurement strategy realizable, and second, thanks to the tunable nature of the PC WG, one can choose to work in the desired wavelength, which is crucial for the choice of QDs and the detectors to be used.

\section{DESIGN}

Before concluding, we will suggest a realizable design with the currently available QDs. Due to the tunable nature of our platform, we have a great freedom in our choice of QDs and the PC WG to be used. Since most detectors operate at $1550 \mathrm{~nm}$, it would be reasonable to choose our QDs and the PC WG such that they also operate in that telecom wavelength regime. Hence, the InP-based PC WG we mentioned, which is achievable in terms of fabrication, is a good candidate for the PC WG, since it can be tuned to yield zero-index behavior at a wavelength of $1550 \mathrm{~nm}$. There exist commercial QDs whose emission/absorption spectra are also within that range. So by exploiting the tunability of the platform we suggested, one can achieve a design operating at the telecom wavelength.

\section{CONCLUSIONS}

In conclusion, we have suggested a novel platform for quantum entanglement measurement, which is both tunable and accessible. Our platform eliminates the difficulty in QD placement by exploiting the ENZ behavior. Moreover, the suggested PC WG structure is also accessible in terms of fabrication and also has the significant feature of tunability. Finally the suggested platform with additional QDs is a strong candidate for quantum error correction schemes. Therefore, the suggested zero-index PC WG platform can open up new horizons in quantum information science.

\section{ASSOCIATED CONTENT}

\section{Supporting Information}

The Supporting Information is available free of charge on the ACS Publications website at DOI: 10.1021/acsphotonics.6b00576.

Derivation of the concurrence and details on numerical calculations (PDF)

\section{AUTHOR INFORMATION}

\section{Corresponding Authors}

*E-mail: ozgune@bilkent.edu.tr.

*E-mail: hcaglayan@bilkent.edu.tr.

\section{Notes}

The authors declare no competing financial interest.

\section{ACKNOWLEDGMENTS}

The authors thank Hodjat Hajian and Mirbek Turduev for their help with the simulations and Ceyhun Bulutay for his comments on the manuscript. This work was supported by Project Nos. TUBITAK-114E505, DPT-HAMIT, NATO-SET193. The authors (E.O. and H.C.) also acknowledge the partial support from the Turkish Academy of Sciences.

\section{REFERENCES}

(1) Nielsen, M. A.; Chuang, I. L. Quantum Computation and Quantum Information; Cambridge Univ. Press, 2000.

(2) Bennett, C. H.; Brassard, G.; Crépeau, C. Teleporting an unknown quantum state via dual classical and Einstein-PodolskyRosen channels. Phys. Rev. Lett. 1993, 70, 1895-1899.

(3) Nölleke, C.; Neuzner, A.; Reiserer, A.; Hahn, C.; Rempe, G.; Ritter, S. Efficient Teleportation Between Remote Single-Atom Quantum Memories. Phys. Rev. Lett. 2013, 110, 140403.

(4) Ekert, A. K. Quantum cryptography based on Bell's theorem. Phys. Rev. Lett. 1991, 67, 661-663.

(5) Knill, E.; Laflamme, R.; Milburn, G. J. A scheme for efficient quantum computation with linear optics. Nature 2001, 409, 46-52.

(6) Wootters, W. K. Entanglement of Formation of an Arbitrary State of Two Qubits. Phys. Rev. Lett. 1998, 80, 2245-2248.

(7) Kim, D.; Carter, S. G.; Greilich, A.; Bracker, A. S.; Gammon, D. Ultrafast optical control of entanglement between two quantum-dot spins. Nat. Phys. 2011, 7, 223-229.

(8) Albert, F.; Sivalertporn, K.; Kasprzak, J.; Strauß, M.; Schneider, C.; Höfling, S.; Kamp, M.; Forchel, A.; Reitzenstein, S.; Muljarov, E. A.; Langbein, W. Microcavity controlled coupling of excitonic qubits. Nat. Commun. 2013, 4, 1747.

(9) DiCarlo, L.; Reed, M. D.; Sun, L.; Johnson, B. R.; Chow, J. M.; Gambetta, J. M.; Frunzio, L.; Girvin, S. M.; Devoret, M. H.; Schoelkopf, R. J. Preparation and measurement of three-qubit entanglement in a superconducting circuit. Nature 2010, 467, 574578 .

(10) Neumann, P.; Mizuochi, N.; Rempp, F.; Hemmer, P.; Watanabe, H.; Yamasaki, S.; Jacques, V.; Gaebel, T.; Jelezko, F.; Wrachtrup, J. Multipartite Entanglement Among Single Spins in Diamond. Science 2008, 320, 1326-1329.

(11) Häffner, H.; Hänsel, W.; Roos, C. F.; Benhelm, J.; Chek-al-kar, D.; Chwalla, M.; Kürber, T.; Rapol, U. D.; Riebe, M.; Schmidt, P. O.; Becher, C.; Gühne, O.; Dür, W.; Blatt, R. Scalable multiparticle entanglement of trapped ions. Nature 2005, 438, 643-646. 
(12) Gao, W.-B.; Lu, C.-Y.; Yao, X.-C.; Xu, P.; Gühne, O.; Goebel, A.; Chen, Y.-A.; Peng, C.-Z.; Chen, Z.-B.; Pan, J.-W. Experimental demonstration of a hyper-entangled ten-qubit Schrödinger cat state. Nat. Phys. 2010, 6, 331-335.

(13) Gonzalez-Tudela, A.; Martin-Cano, D.; Moreno, E.; MartinMoreno, L.; Tejedor, C.; Garcia-Vidal, F. J. Entanglement of Two Qubits Mediated by One-Dimensional Plasmonic Waveguides. Phys. Rev. Lett. 2011, 106, 020501.

(14) Cano, D. M.; Tudela, A. G.; Moreno, L. M.; Vidal, F. J. G.; Tejedor, C.; Moreno, E. Dissipation-driven generation of two-qubit entanglement mediated by plasmonic waveguides. Phys. Rev. B: Condens. Matter Mater. Phys. 2011, 84, 235306.

(15) Zheng, H.; Baranger, H. U. Persistent Quantum Beats and Long-Distance Entanglement from Waveguide-Mediated Interactions. Phys. Rev. Lett. 2013, 110, 113601.

(16) Liao, Z.; Zeng, X.; Zhu, S.- Y.; Zubairy, M. S. Single-photon transport through an atomic chain coupled to a one-dimensional nanophotonic waveguide. Phys. Rev. A: At., Mol., Opt. Phys. 2015, 92, 023806.

(17) Loss, D.; DiVincenzo, D. P. Quantum computation with quantum dots. Phys. Rev. A: At., Mol., Opt. Phys. 1998, 57, 120-126.

(18) Jin, X. R.; Sun, L.; Yang, X.; Gao, J. Quantum entanglement in plasmonic waveguides with near-zero mode indices. Opt. Lett. 2013, 38, 4078-4081.

(19) Ballestero, C. G.; Moreno, E.; Vidal, F. J. G. Generation, manipulation, and detection of two-qubit entanglement in waveguide QED. Phys. Rev. A: At., Mol., Opt. Phys. 2014, 89, 042328.

(20) Angelatos, G.; Hughes, S. Entanglement dynamics and Mollow nonuplets between two coupled quantum dots in a nanowire photonic-crystal system. Phys. Rev. A: At., Mol., Opt. Phys. 2015, 91, 051803.

(21) Angelatos, G.; Hughes, S. Polariton waveguides from a quantum dot chain in a photonic crystal waveguide: an architecture for waveguide quantum electrodynamics. Optica 2016, 3, 370-376.

(22) Silveirinha, M. G.; Engheta, N. Tunneling of Electromagnetic Energy through Subwavelength Channels and Bends using $\varepsilon$-NearZero Materials. Phys. Rev. Lett. 2006, 97, 157403.

(23) Engheta, N. Pursuing Near-Zero Response. Science 2013, 340, $286-287$.

(24) Edwards, B.; Alù, A.; Young, M. E.; Silveirinha, M.; Engheta, N. Experimental Verification of Epsilon-Near-Zero Metamaterial Coupling and Energy Squeezing Using a Microwave Waveguide. Phys. Rev. Lett. 2008, 100, 033903.

(25) Smolyaninov, I. I.; Smolyaninova, V. N.; Kildishev, A. V.; Shalaev, V. M. Anisotropic Metamaterials Emulated by Tapered Waveguides: Application to Optical Cloaking. Phys. Rev. Lett. 2009, 102, 213901.

(26) Peropadre, B.; Zueco, D.; Porras, D.; Ripoll, J. J. G. Nonequilibrium and Nonperturbative Dynamics of Ultrastrong Coupling in Open Lines. Phys. Rev. Lett. 2013, 111, 243602.

(27) Vesseur, E. J. R.; Coenen, T.; Caglayan, H.; Engheta, N.; Polman, A. Experimental Verification of $\mathrm{n}=0$ Structures for Visible Light. Phys. Rev. Lett. 2013, 110, 013902.

(28) Alù, A.; Bilotti, F.; Engheta, N.; Vegni, L. Metamaterial covers over a small aperture. IEEE Trans. Antennas Propag. 2006, 54, 16321643.

(29) Adams, D. C.; Inampudi, S.; Ribaudo, T.; Slocum, D.; Vangala, S.; Kuhta, N. A.; Goodhue, W. D.; Podolskiy, V. A.; Wasserman, D. Funneling Light through a Subwavelength Aperture with EpsilonNear-Zero Materials. Phys. Rev. Lett. 2011, 107, 133901.

(30) Wang, B.; Huang, K.-M. Shaping the radiation pattern with mu and epsilon-near-zero metamaterials. Prog. Electromagn. Res. 2010, 106, 107-119.

(31) Pena, V. P.; Torres, V.; Cia, M. N.; Beruete, M.; Sorolla, M.; Engheta, N. $\varepsilon$-Near-Zero Graded Index Structure as a Bi-concave Metallic Lens Using Stacked Rectangular near Cut-off Waveguides; 7th European Conference on Antennas and Propagation (EuCAP) 2535 Gothenburg, Sweden, April 8-12, 2013. .
(32) Yoshie, T.; Vučković, J.; Scherer, A.; Chen, H.; Deppe, D. High quality two-dimensional photonic crystal slab cavities. Appl. Phys. Lett. 2001, 79, 4289-4291.

(33) Cubukcu, E.; Aydin, K.; Ozbay, E.; Foteinopolou, S.; Soukoulis, C. Electromagnetic waves: Negative Refraction by Photonic Crystals. Nature 2003, 423, 604-605.

(34) Turduev, M.; Botey, M.; Giden, I.; Herrero, R.; Kurt, H.; Ozbay, E.; Staliunas, K. Two-dimensional complex parity-time-symmetric photonic structures. Phys. Rev. A: At., Mol., Opt. Phys. 2015, 91, 023825 .

(35) Serebryannikov, A. E.; Cakmak, A. O.; Colak, E.; Caglayan, H.; Kurt, H.; Ozbay, E. Multiple slow waves and relevant transverse transmission and confinement in chirped photonic crystals. Opt. Express 2014, 22, 21806.

(36) Kok, A. A. M.; van Heesch, C. M.; Geluk, E. J.; Sander-Jochem, M. J. H.; van der Tol, J. J. G. M.; Oei, Y. S.; Smit, M. K. Twodimensional photonic crystals based on InP rods. Proc. IEEE/LEOS Symp. (Benelux Chapter) Mons, Belgium 2005, 273.

(37) Shen, J. T.; Fan, S. Coherent photon transport from spontaneous emission in one-dimensional waveguides. Opt. Lett. 2005, 30, 2001-2003.

(38) Shen, J. T.; Fan, S. Coherent Single Photon Transport in a OneDimensional Waveguide Coupled with Superconducting Quantum Bits. Phys. Rev. Lett. 2005, 95, 213001.

(39) Shen, J. T.; Fan, S. Strongly Correlated Two-Photon Transport in a One-Dimensional Waveguide Coupled to a Two-Level System. Phys. Rev. Lett. 2007, 98, 153003.

(40) Shen, J. T.; Fan, S. Theory of single-photon transport in a single-mode waveguide. I. Coupling to a cavity containing a two-level atom. Phys. Rev. A: At., Mol., Opt. Phys. 2009, 79, 023837. 